\title{
Decursin inhibits growth of human bladder and colon cancer cells via apoptosis, G1-phase cell cycle arrest and extracellular signal-regulated kinase activation
}

\author{
WUN-JAE KIM ${ }^{1,3}$, SE-JUNG LEE ${ }^{2,3}$, YOUNG DEUK CHOI ${ }^{4}$ and SUNG-KWON MOON ${ }^{2,3}$ \\ ${ }^{1}$ Department of Urology, Chungbuk National University College of Medicine, Cheongju, Chungbuk 361-763; \\ ${ }^{2}$ Department of Food and Biotechnology, Chungju National University, Chungju, Chungbuk 380-702; \\ ${ }^{3}$ Personalized Tumor Engineering Research Center, Chungbuk National University, Cheongju, Chungbuk 361-763; \\ ${ }^{4}$ Department of Urology, Yonsei University College of Medicine, Seoul, Korea
}

Received October 19, 2009; Accepted December 21, 2009

DOI: 10.3892/ijmm_00000386

\begin{abstract}
Decursin, a pyranocoumarin isolated from the Korean Angelica gigas root, has demonstrated anti-cancer properties. In the present study, we found that decursin inhibited cell viability in cultured human urinary bladder cancer $235 \mathrm{~J}$ cells and colon cancer HCT116 cells. The inhibited proliferation was due to apoptotic induction, because both cells treated with decursin dose-dependently showed a sub-G1 phase accumulation and an increased cytoplasmic DNA-histone complex. Cell death caused by decursin was also associated with the downregulation of anti-apoptotic factor Bcl-2 and the up-regulation of pro-apoptotic molecules cytochrome c, caspase 3 and Bax. Treatment of both types of cancer cells with decursin resulted in G1-phase cell cycle arrest, as revealed by FACS analyses. In addition, decursin increased protein levels of p21WAF1 with a decrease in cyclins and cyclin dependent kinases (CDKs). Furthermore, decursin induced the activation of extracellular signal-regulated kinases (ERK) in both cancer cell lines, with the notable exceptions of c-Jun N-terminal kinase (JNK) and p38 mitogen activated protein (MAP) kinase. Finally, pretreatment with ERK-specific inhibitor PD98059 reversed decursin-induced p21WAF1 expression and decursin-inhibited cell growth. Thus, these findings suggest that decursin has potential therapeutic efficacy for the treatment of bladder and colon cancer.
\end{abstract}

\section{Introduction}

In the past several decades, the occurrence of malignant cancer has increased. For example, colon cancer is the one of the most

Correspondence to: Dr Sung-Kwon Moon, Department of Food and Biotechnology, Chungju National University, 123 Geomdan-ri Iryu-myeon, Chungju, Chungbuk 380-702, Korea

E-mail:skmoon@cjnu.ac.kr

Key words: decursin, bladder cancer cells, colon cancer cells, G1-phase cell cycle arrest, apoptosis, p21WAF1, extracellular signalregulated kinases common malignancies in the world (1), and bladder cancer is the second most common cancer of the genitourinary tract worldwide (2). Although pharmacological studies have led to a marked improvement in treatment and diagnosis at early stages, no effective therapy is yet available for prognosis. Therefore, there is a great need for the development of new therapeutic strategies for the treatment of cancer. One approach, as pursued in the present study, is to increase efforts to isolate bioactive materials from medicinal plants that are capable of activating the cellular apoptotic response, retarding the cell cycle, and regulating the signaling pathway in cancerous cells.

Decursin is a major active compound found in the roots of Angelica gigas Nakai, which has been traditionally used to treat female afflictions and anemia $(3,4)$. There are some reports about the pharmacological effects of this compound showing anti-bacterial and anti-leukemic activity, activation of protein kinase $C$, an and anti-angiogenic effect (3-7). Decursin reportedly inhibits growth and promotes apoptosis in several other cancer cells $(8,9)$. However, there has been no report of the relationship between cell cycle regulation and the extracellular signal-regulated kinases (ERK) activation in impairing the growth of cancer cells.

The present study is the first to establish the efficacy of decursin in the treatment of colon and bladder cancer cells. We found that decursin significantly inhibited cell growth and caused apoptosis in both cell lines. In addition, we investigated the mechanistic rationale for the observed efficacy of decursin, which showed a link between cell cycle modulation and ERK activation.

\section{Materials and methods}

Materials. Polyclonal antibodies to caspase 3, Bax, Bcl-2, and cytochrome c were obtained from, cyclin E, cyclin dependent kinases (CDK) 2 and CDK4 were obtained from Santa Cruz Biotechnology (Santa Cruz, CA, USA). Polyclonal antibodies to ERK, phospho-ERK, p38 MAP kinase, phospho-p38 MAP kinase, c-Jun N-terminal kinases (JNK) and phospho-JNK were obtained from Cell signaling (Beverly, MA). Polyclonal antibodies to cyclin D1, p21WAF1 (556430), p27 (554069), and 
p53 were obtained from BD Pharmingen (San Jose, CA). PD98059 was obtained from Calbiochem (San Diego, CA).

Preparation of decursin. The root of Angelica gigas was purchased in a local Oriental medicine market in Seoul, Korea in 2006. The plant materials were authenticated by an expert at Chungbuk National University. The extraction and fractionation of air-dried powdered root was prepared as described previously (9). Silica gel column chromatography was used to isolate decursin (9). These compounds were characterized by nuclear magnetic resonance (Bruker Avance 400 NMR spectrometer) and mass spectroscopy (by Jeol JMS-AX505-WA mass spectrometer) at Natural Product Research Institute, Seoul National University, Seoul, Korea. The purified coumarin compound, decursin, was dissolved in DMSO as stock solutions, and used directly in cell culture treatments.

Cell cultures. The human bladder carcinoma cell line (253J) and human colon cancer cell line (HCT116) were obtained from the American Type Culture Collection. Cells were maintained in DMEM (4.5 g glucose/l) supplemented with $10 \%$ fetal calf serum, L-glutamine, and antibiotics (Biological Industries, Beit Haemek, Israel) at $37^{\circ} \mathrm{C}$ in a $5 \% \mathrm{CO}_{2}$ humidified incubator.

Cell viability assay. Subconfluent 5637 cells, exponentially growing in 24-well plates, were incubated with cordycepin for various lengths of time. Cell viability was determined using a modification of the 3-(4,5-dimethylthiazol-2-yl)-2,5diphenyltetrazolium bromide (MTT) assay, which is based on the conversion of tetrazolium salt 3-(4,5-dimethylthiazol-2yl)-5-(3-carboxymethoxyphenyl)-2-(4-sulfophenyl)-2-tetrazolium to the formazan product by mitochondrial dehydrogenase. The formazan product was quantified by measurement of absorbance at $490 \mathrm{~nm}(10)$.

DAPI staining. After decursin treatment for $24 \mathrm{~h}$, cellular morphology was observed under an inverted light microscope. Moreover, the treated cells were fixed with ice-cold $4 \%$ paraformaldehyde at $4{ }^{\circ} \mathrm{C}$ for $20 \mathrm{~min}$, and washed with ice-cold PBS, then permeabilized with $0.3 \%$ Triton X-100. After being washed twice with PBS, the treated cells were stained with fluorochrome dye 4'6-diamidino-2-phenylindole (DAPI, Santa Cruz, USA). The cells were observed under a fluorescence microscope with a peak excitation wavelength of $340 \mathrm{~nm}$.

Apoptosis detection by ELISA. This method is based on a quantification of the enrichment of mono- and oligonucleosomes in the cytoplasm using a Cell Death Detection ELISA kit (Roche, Mannheim, Germany) (10).

Cell cycle analysis (FACS). Cells were harvested, fixed in $70 \%$ ethanol, and stored at $-20^{\circ} \mathrm{C}$. Cells were then washed twice with ice-cold PBS and incubated with RNase and the DNA intercalating dye, propidium iodide. Cell cycle phase analysis was performed using Becton Dickinson Facstar flow cytometer equipped with Becton Dickinson cell fit software.

For flow cytometric analysis, a FACSCalibur flow cytometer (Becton Dickinson, NJ, USA) equipped with a single argon ion laser was used. The excitation wavelength was $488 \mathrm{~nm}$, and the emission filters were 515-545 BP, 572-588 BP
A
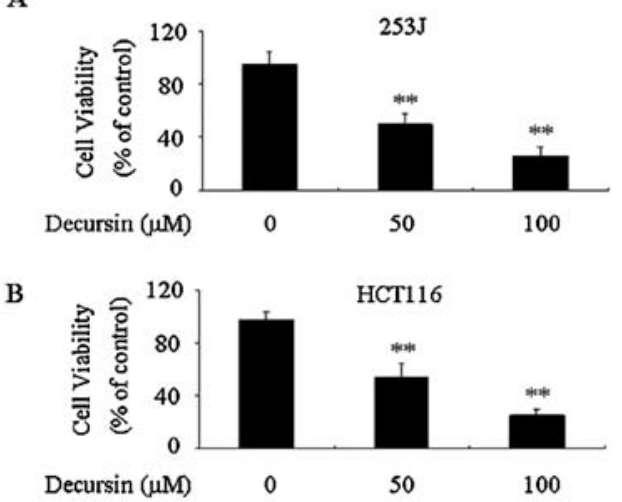

Figure 1. Effects of decursin on cell viability in 253J (A) and HCT116 (B) cells. Subconfluent exponentially growing cells were incubated with decursin for $24 \mathrm{~h}$ at the indicated concentrations in $10 \%$ DMEM. Cell viability was determined using a modified MTT assay. Results are presented as means $\pm \mathrm{SE}$ from three triplicate experiments. ${ }^{* *} \mathrm{P}<0.01$ compared with no decursin treatment.

and 600 LP. Forward light scatter, which was correlated with the size of the cell, and right-angle light scatter, which was correlated with the complexity of the cytoplasm, were used to establish size gates and exclude cellular debris from the analysis. The DNA content of 10,000 cells per analysis was monitored using the FACSCalibur system. DNA fluorescence of PI-stained cells was evaluated by excitation at $488 \mathrm{~nm}$ and by monitoring through a $630 / 22 \mathrm{~nm}$ band pass filter. A minimum of 10,000 cells per sample was used for analysis, which was performed using CellQuest software. Apoptotic nuclei were identified as a subploid DNA peak, and were distinguished from cell debris based on forward light scatter and PI fluorescence. Representative flow cytometry patterns are shown.

Immunoprecipitation, immunoblotting, and immune complex kinase assays. Cells were treated with cordycepin in the presence of $10 \% \mathrm{FBS}$ at $37^{\circ} \mathrm{C}$ for different durations. Cell lysates were prepared, and immunoprecipitation, immunoblotting and immune complex kinase assays were performed as described previously (10).

Statistical analysis. When appropriate, data were expressed as means \pm SE. Data were analyzed by factorial ANOVA and Fisher's least significant difference test where appropriate. Statistical significance was set at $\mathrm{P}<0.01$.

\section{Results}

Decursin inhibits the proliferation of two different kinds of human cancer cells. To determine the anti-proliferative effect of decursin on 235J bladder cancer cells and HCT116 colon cancer cells, both cell lines were cultured for $24 \mathrm{~h}$ with or without various concentrations of decursin $(0-100 \mu \mathrm{M})$. Decursin treatment showed a strong dose-dependent inhibition of cell growth in 235J and HCT116 cells (Fig. 1A and B).

Decursin induced apoptosis in 235J and HCT116 cells. Further experiments were carried out to determine if the inhibitory 
$253 \mathrm{~J}$
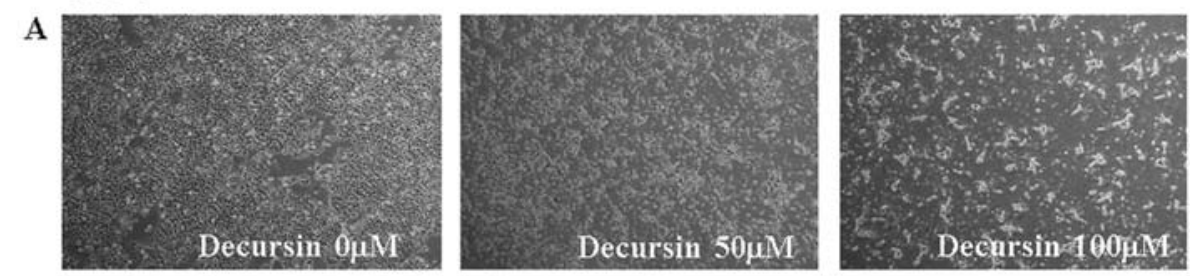

B
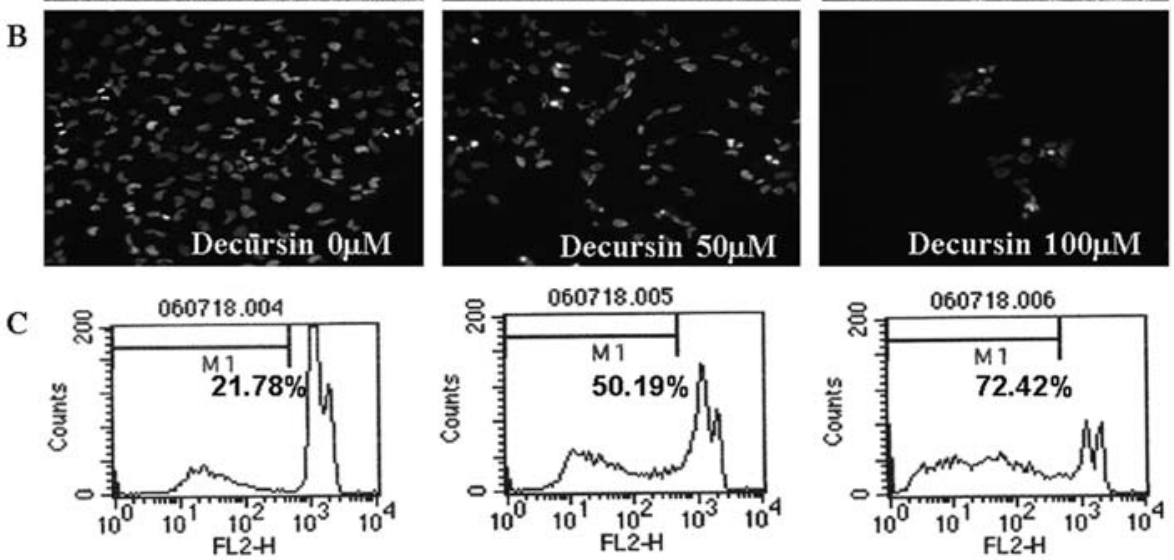

Figure 2. Induction of apoptosis by decursin in $253 \mathrm{~J}$ cells. (A) Phase contrast micrographs of decursin-treated $253 \mathrm{~J}$ cells. Cells were treated with 0,50 , or $100 \mu \mathrm{M}$ of decursin for $24 \mathrm{~h}$. A typical result from three independent experiments is shown. (B) Fluoresence micrographs by staining with DAPI. (C) The percentage of cells in the sub-G1 fraction was increased with decursin. After being treated with decursin $(0,50$, or $100 \mu \mathrm{M})$ for $24 \mathrm{~h}$, the DNA content in cells was assessed by flow cytometry.

HCT116

A
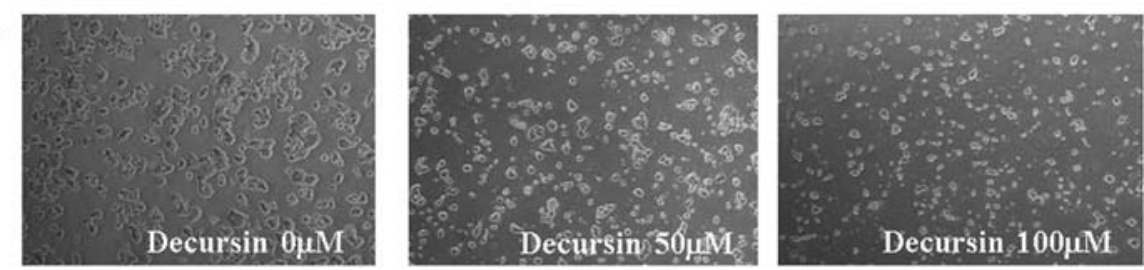

B
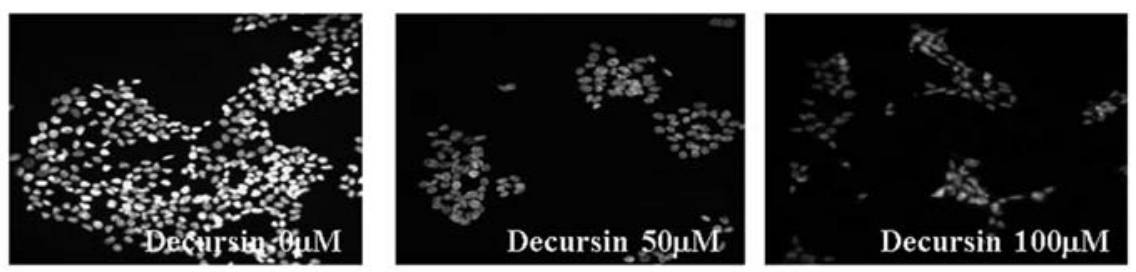

c

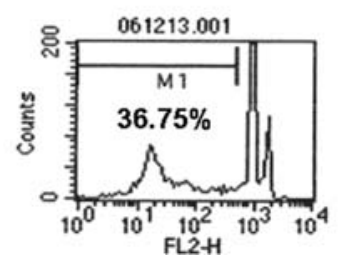

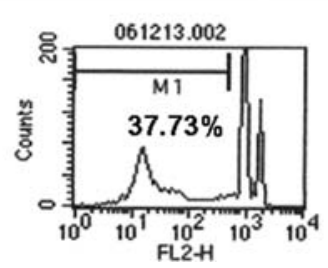

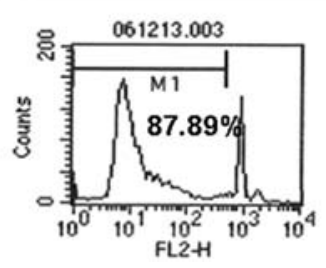

Figure 3. Decursin-induced apoptosis in HCT116 cells. HCT116 cells were treated with 0, 50, or $100 \mu \mathrm{M}$ decursin for 24 h. (A) Morphological changes of cells were examined under phase contrast micrograph. (B) After cells were treated with decursin at the indicated concentrations $(0,50$, or $100 \mu \mathrm{M})$ for $24 \mathrm{~h}$, the cells were fixed, stained with DAPI, and the nuclear morphology was then photographed under fluoresence micrograph. (C) Decursin increased the sub-G1 fraction in HCT116 cells. Cells were treated with decursin $(0,50$, or $100 \mu \mathrm{M})$, followed by cell fixation. Then, cells were stained with PI, and DNA content in cells was analyzed by flow cytometry.

effect of decursin on the cell proliferation is due to the result of apoptotic cell death. Direct observation detected by phasecontrast microscopy showed that exposure to decursin was associated with cell shrinkage and cytoplasm condensation in 235J and HCT116 cells (Figs. 2A and 3A). In addition, nuclei with chromatin condensation and the formation of apoptotic bodies were observed by DAPI staining in both types of cancer cells cultured with decursin (Figs. 2B and 3B). We next quantified the amount of sub-G1 DNA population after treatment with decursin in both types of cancer cells using flow cytometry analysis. Compared with the control, $100 \mu \mathrm{M}$ of decursin increased the percentage of cells in the sub-G1 
A

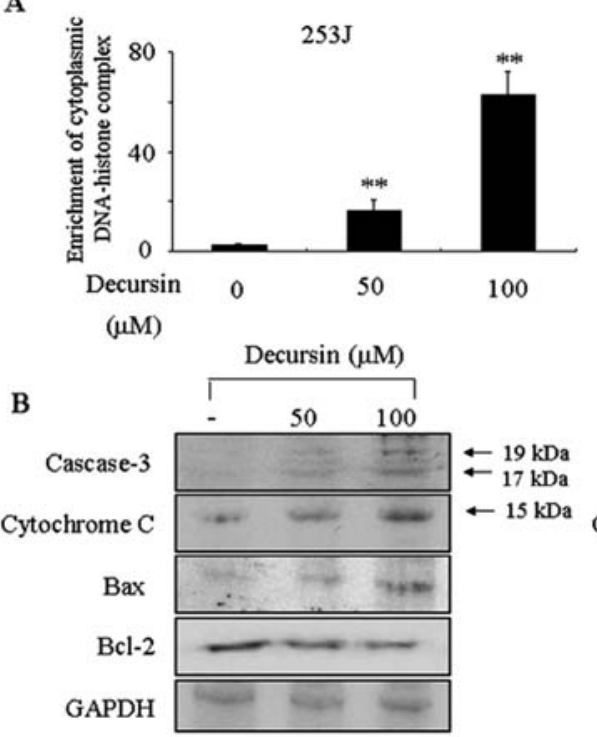

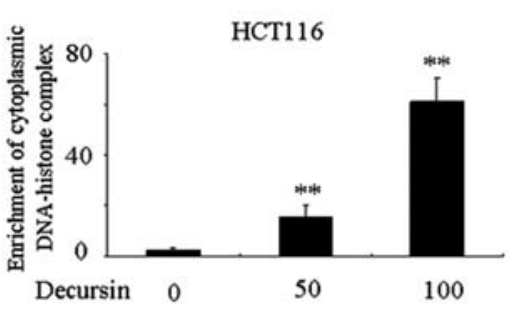

$(\mu \mathrm{M})$

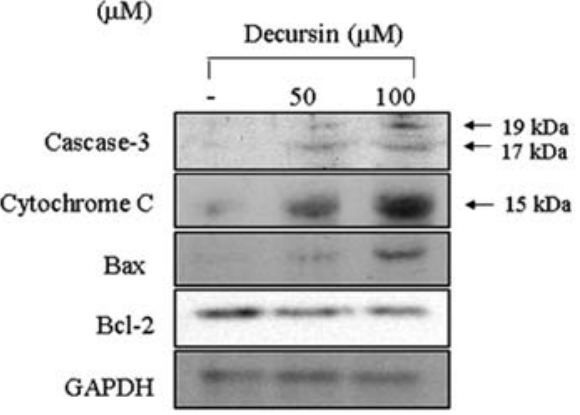

Figure 4. Decursin-mediated apoptosis is associated with formation of cytoplasmic DNA-histone complex and expression of caspase 3, cytochrome c, Bax and Bcl-2. (A) Both cells were treated with decursin, and cultured for $24 \mathrm{~h}$. The cytoplasmic DNA-histone complex was measured by ELISA. Results are presented as means $\pm \mathrm{SE}$ from three triplicate experiments. ${ }^{* *} \mathrm{P}<0.01$ compared with no decursin treatment. (B) Both cell lines were treated with decursin $(0$, 50 , or $100 \mu \mathrm{M}$ ) for $24 \mathrm{~h}$. The expression of caspase 3, cytochrome c, Bax and Bcl-2 was analyzed by immunoblot analysis. The results from representative experiments were normalized to GAPDH expression.

A

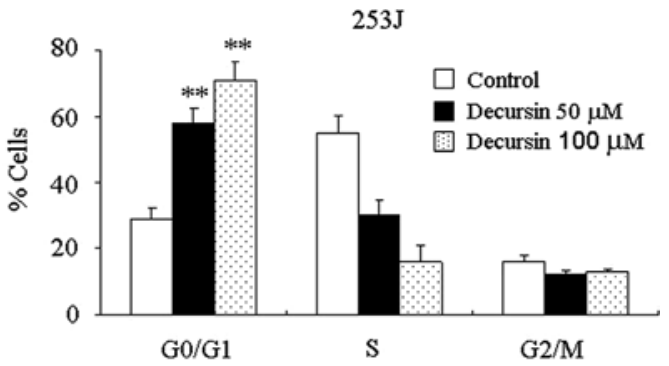

B

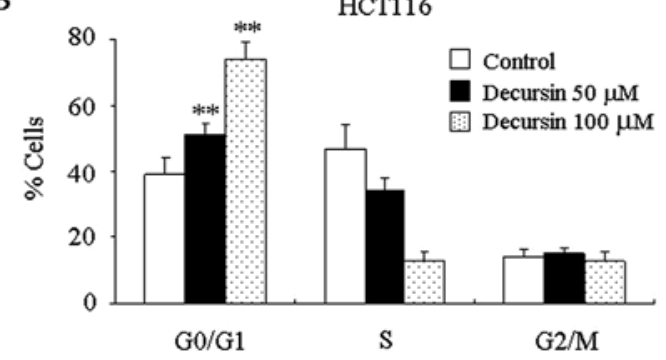

Figure 5. Decursin induces G1-phase cell cycle arrest in 253J and HCT116 cells. 253J (A) and HCT116 (B) cells were treated with 0,50 , or $100 \mu \mathrm{M}$ decursin for $24 \mathrm{~h}$. Cells were subjected to flow cytometric analysis to examine the effect of decursin on cell cycle distribution. The percentage of cells in each population is presented. The data are represented as the mean $\pm \mathrm{SE}$ of three experiments performed in triplicate. ${ }^{* *} \mathrm{P}<0.01$ compared with no decursin treatment.

fraction from 21 to $72 \%$ and from 36 to $87 \%$ in $235 \mathrm{~J}$ and HCT116 cells, respectively (Figs. 2C and 3C). Additionally, ELISA-based assay (see Materials and methods) was examined to detect histone-associated oligonucleosome DNA fragments in decursin-treated cells. As shown in Fig. 4A, decursin significantly increased the cytoplasmic DNA-histone complex in both types of cancer cells. These results show that there was a significant increase in apoptosis in cancer cell lines exposed to decursin.

Decursin-induced apoptosis was associated with the upregulation of caspase 3, cytochrome c, and Bax and the downregulation of Bcl-2. To further analyze the role of decursin in the inducement of apoptosis, the expressions of caspase 3, cytochrome c, Bax and Bcl-2 were tested in both types of cancer cells after treatment with different concentrations of decursin. Immunoblotting analysis showed that the expressions of caspase 3, cytochrome $\mathrm{c}$ and Bax were increased in both types of decursin-treated cancer cells (Fig. 4B), whereas the levels of Bcl-2 protein were down-regulated (Fig. 4B).

Decursin induced G1-phase cell cycle arrest in 235J and HCT116 cells. Flow cytometric analysis was performed to examine whether decursin treatment of both types of cancer cells resulted in cell cycle arrest at a specific point in the cell cycle progression. Upon exposure to decursin (50-100 $\mu \mathrm{M}$ ), analysis of the cell cycle distribution showed that these cells had marked accumulation in the G1 phase of the cell cycle (Fig. 5A and B). We next examined the expression of cell cycle regulatory factors at the G1 boundary, such as cyclin D1, cyclin E, CDK2, and CDK4, in both types of cancer cells treated with decursin using immunoblot and kinase assays. As shown in Fig. 6A, compared with control, decursin treatment for $24 \mathrm{~h}$ showed a dose-dependent decrease in protein levels of cyclin D1, cyclin E, CDK2, and CDK4. In addition, the CDK2- and CDK4-associated kinase activities were reduced after treating both types of cancer cells with decursin (Fig. 6B).

Induction of CDK inhibitor p21WAF1 by decursin. Next, to examine whether CDK inhibitors are involved in the decursinmediated inhibition of cell growth in 235J and HCT116 cells, 


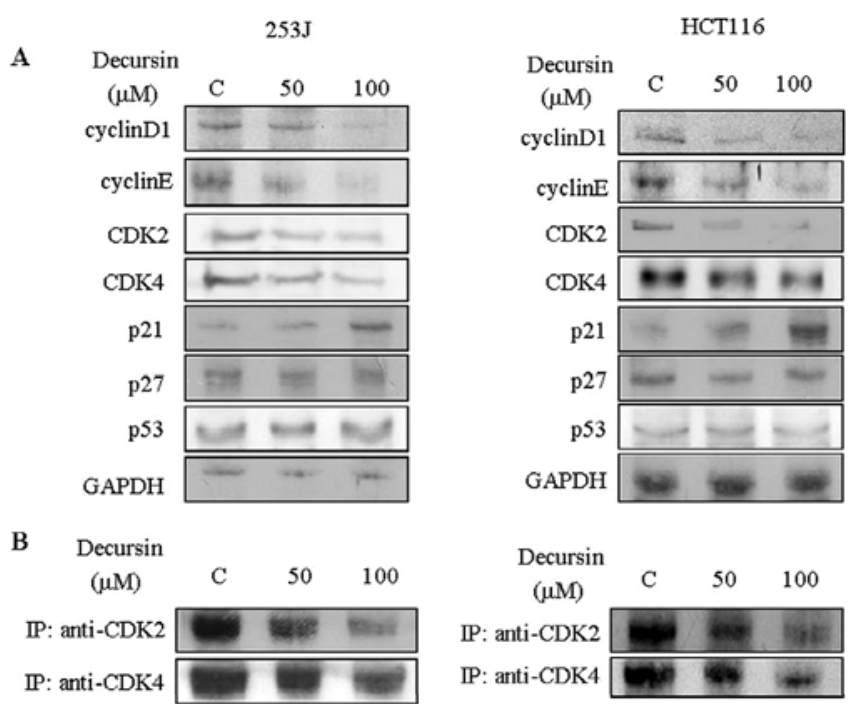

Figure 6. Effect of decursin on G1-phase cell cycle regulators and CDKs kinase activity in 253J and HCT116 cells. (A) Both cell lines were cultured in $10 \%$ serum-contained medium, and treated with decursin at the indicated concentrations. Total cell lysates were prepared and subjected to immunoblot analysis, which was performed with antibodies specific for cyclin D1, cyclin E, CDK2, CDK4, p21WAF1, p27 and p53. The results from representative experiments were normalized to GAPDH expression. (B) Equal amounts of cell lysates were prepared and immunoprecipitated with anti-CDK2 and -CDK4 antibodies. The kinase reaction was performed using histone $\mathrm{H} 1$ (for CDK2) or GST-Rb (for CDK4) as substrates.

the levels of these proteins were analyzed by immunoblot. Treatment of $235 \mathrm{~J}$ cells with decursin resulted in a dosedependent increase in p21WAF1 expression, compared with untreated cells (Fig. 6A). However, decursin treatment did not affect the expression of either p27 or p53 tumor suppressor proteins (Fig. 6A). In addition, similar results were obtained in HCT116 cells under the same experimental conditions (Fig. 6A).

Decursin induced ERK activation in 235J and HCT116 cells. We attempted to determine whether decursin affects activation of the MAPK, including ERK1/2, JNK and p38 MAP kinase. Treatment with decursin resulted in the activation of ERK in both 235J and HCT116 cell lines (Fig. 7A). Pretreatment with PD98059, a specific ERK inhibitor, blocked the decursininduced activation of ERK in both cell lines (Fig. 7B). However, decursin treatment did not affect JNK and p38 MAP kinase activation (Fig. 7A). The results of these experiments suggest that ERK activation is important in decursin-mediated inhibition of cell growth.

Inhibition of ERK activation blocked decursin-induced p21WAF1 expression and cell growth inhibition. To further assess the biological significance of ERK activation in mediating decursin-induced p21WAF1 expression, the alteration of $\mathrm{p} 21 \mathrm{WAF} 1$ induction on the ERK signaling pathway was investigated. Thus, 235J and HCT116 cells were pretreated for $40 \mathrm{~min}$ with or without the ERK upstream kinase MEK1/2 inhibitor PD98059, which was followed by exposure to decursin. PD98059 blocked decursin-induced p21WAF1 expression in both types of cancer cells (Fig. 8A). We next investigated the relevance of ERK activation in
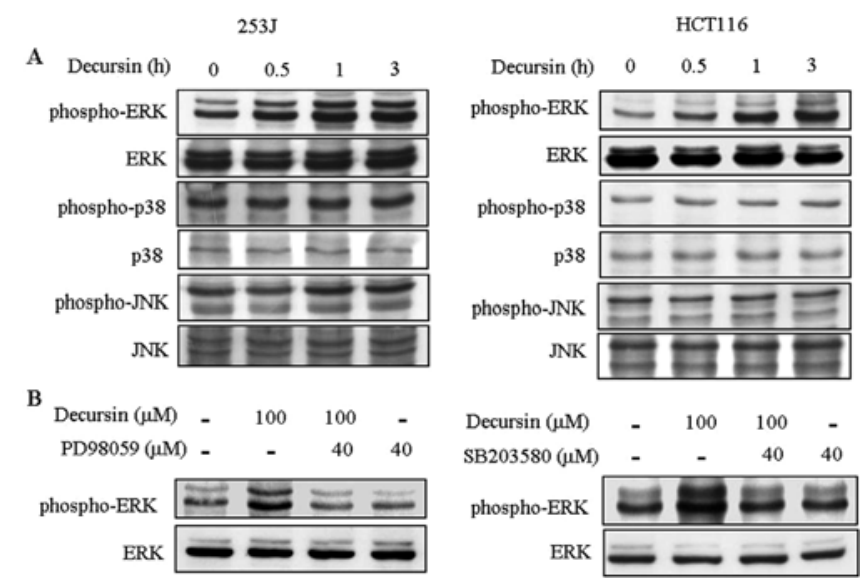

Figure 7. Decursin induced ERK1/2 activation in 253J and HCT116 cells. (A) Both cells were treated with decursin $(100 \mu \mathrm{M})$ at the indicated times. Cell lysates were prepared, and the phosphorylation levels of ERK1/2, JNK and p38 MAP kinase were detected by immunoblot analysis using antibodies phospho-specific for ERK1/2, JNK and p38 MAP kinase. (B) Both types of cells were pretreated for $40 \mathrm{~min}$ with PD98059 (40 (M), and then both types were treated with decursin $(100 \mu \mathrm{M})$ for $3 \mathrm{~h}$. The level of phospho-ERK1/2 was detected using immunoblot analysis.

decursin-induced inhibition of cell growth. When the 235J and HCT116 cells were pretreated with PD98059, inhibition of cell growth in response to decursin markedly attenuated (Fig. 8B). These results indicate that ERK activation must be involved in mediation of the p21WAF1 expression and growth inhibition in bladder and colon cancer cells induced by decursin treatment.

\section{Discussion}

Many studies have been undertaken to elucidate the anticancer effects of decursin on apoptosis induction and inhibition of cell growth in several cancer cell lines $(8,9)$. However, the molecular mechanism by which decursin inhibits cell proliferation, including cross-talk between the cell cycle and the signaling pathway, is not yet understood. The present study is the first to show that decursin-induced G1 cell cycle arrest via p21WAF1 induction is due to ERK activation in 235J bladder cancer and HCT16 colon cancer cells.

Most of the presently available anti-cancer drugs have the ability to induce tumor cell apoptosis (11). Consistent with this approach, we found that decursin-induced apoptosis in 235J and HCT116 cells is associated with reduced cell viability, an increase of sub-G1 phase cell number, and an increase of cytoplasmic DNA-histone complex in cells. This study also defined the mechanical events of apoptosis that are associated with decursin-induced inhibition of cell growth in both cells. In the cells undergoing apoptosis, cytochrome $\mathrm{c}$ is released from the mitochondria to the cytosol (12). In the cytosol, cytochrome c can activate the caspase-3, cysteine protease $32 \mathrm{kDa}$ proenzyme, CPP32 (13). After caspase-3 activation, the proteolytic degradation of poly(ADP-ribose) polymerase is cleaved, which leads to apoptosis $(14,15)$. In the present study, we demonstrated that decursin-induced apoptotic death in bladder and colon cancer cells is mediated, at least in part, through an increased cytochrome c release and by the activation of caspase-3. 
A

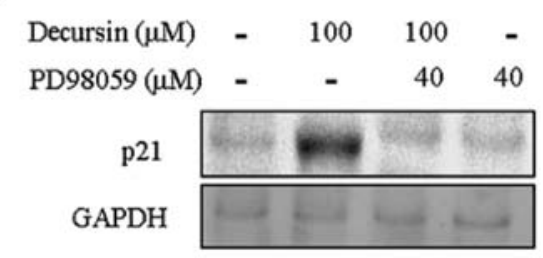

B

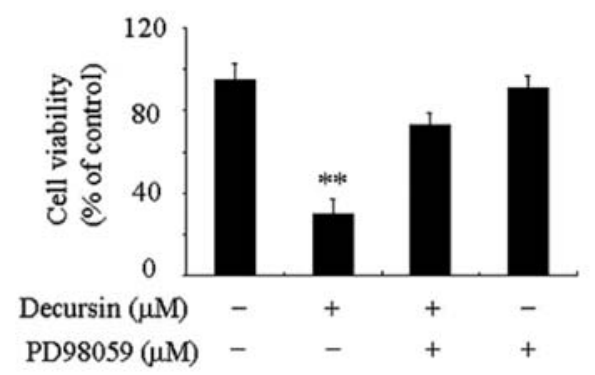

HCT116
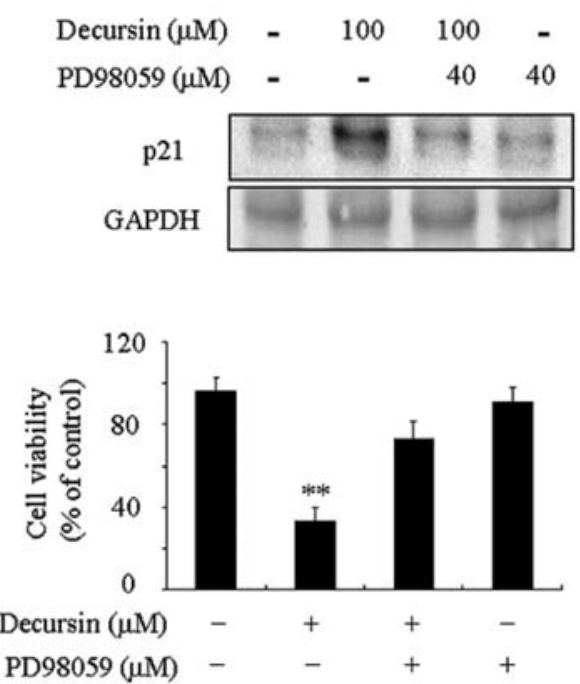

Figure 8. Effect of MEK1/2 inhibitor on decursin-induced p21WAF1 expression and growth inhibition. (A) Cells were pre-incubated for 40 min in the absence or presence of PD98059 $(40 \mu \mathrm{M})$. Cells were then treated with $100 \mu \mathrm{M}$ decursin. Immunoblot analysis was performed with antibodies specific for p21WAF1. The results from representative experiments were normalized to GAPDH expression. (B) Cells were pretreated for 40 min with $40 \mu \mathrm{M}$ PD98059 before cells were treated with $100 \mu \mathrm{M}$ decursin at $24 \mathrm{~h}$. Cell viability was determined by MTT assay. Indicated values are means of triplicate wells. ${ }^{* *} \mathrm{P}<0.01$ compared with no decursin treatment.

Apoptosis is also tightly regulated by a series of Bcl-2 family genes, which are classified into two main categories, Bax (pro-apoptotic protein) and Bcl-2 (anti-apoptotic protein) (16-19). Bcl-2 has proven to have the ability to prevent cytochrome c release from the mitochondria, whereas Bax plays a crucial role in the cell-death machinery, which triggers the release of cytochrome c (20-22). We found that decursin induced apoptosis through increased expression of Bax protein and reduced Bcl-2 protein levels in both types of decursin-treated cells. Results of the present study clearly show that decursin induced cell death-signaling events leading to mitochondrion-related apoptotic pathways, which is closely associated with a loss of mitochondrial membrane permeability and the subsequent release of various apoptosis signaling factors (23).

Cell cycle progression is regulated by cyclin dependent kinases (CDKs), cyclin dependent kinase inhibitors (CDKIs), and cyclins (24-27). CDKI, such as p21WAF1, binds to the active CDK-cyclin complexes leading to down-regulation of the cell cycle progression and thereby inhibiting their kinase activities $(26,27)$. Previous studies reported that decursin induced G1 cell cycle arrest in different cancer cells $(8,9)$. One paper reported p21WAF1 induction and G1 cell cycle arrest in human prostate cancer cells following decursin treatment (9). Consistent with the results of previous studies, the present study showed that decursin induced significant and dosedependent G1-phase cell cycle arrest in bladder and colon cancer cells. Moreover, decursin treatment resulted in a simultaneous decrease in $\mathrm{G} 1$ cell cycle related proteins, including cyclin D1/CDK4 and cyclin E/CDK2. Further investigation showed significant p21WAF1 expression during decursin-induced G1 phase arrest in cancer cells. However, decursin showed unchanged levels in the expression of p27 and p53. These results suggest that increased p21WAF1 expression has a direct relevance in regression of bladder and colon cancer cells induced by decursin, independent of the p53 pathway.

The mitogen activated protein kinase (MAPK) signaling pathway is one of the critical molecular events regulating cell proliferation and migration (28-31). Previous studies have demonstrated that the MAPK pathway plays an important role in anti-proliferative effects and/or regulates the cell cycle under stressful conditions $(29,31)$. MAPKs include extracellular signal-regulated kinases (ERKs), c-Jun N-terminal kinases (JNK, also called stress-activated protein kinase) and p38 MAP kinases (29). The results of the present study indicate that decursin induces ERK activation in bladder and colon cancer cells. These results are inconsistent with previous study results indicating that decursin is associated with decreased activation of ERK in breast cancer and HUVEC cells $(7,8)$. The present study is the first to report the activation of ERK in decursin-induced cancer cell growth inhibition. Many studies have analyzed the effects of decursin on tumorgrowth inhibition in several cell lines $(8,9)$. Recently, the involvement of p21WAF1 has been demonstrated for the regulation of cell growth inhibition in prostate cancer cell lines (9). However, the issue of how the activation of signaling pathways results in cell growth inhibition and induction of p21WAF1 is unclear. In the present study, we identified the ERK-mediated control of the cell growth inhibition in cancer cells in response to decursin. Inhibition of ERK by pretreatment with the pharmacological inhibitor PD98059 attenuated decursin-induced p21WAF1 expression (Fig. 8A). In addition, blockage of ERK with PD98059 prompted a resumption of the cell growth inhibition in cells that was induced by decursin, suggesting that ERK is involved in decursin-induced inhibition of bladder and colon cancer cell growth. The results acquired from the inhibition of ERK activation have provided the first evidence that ERK activation plays a key role in the decursin- 
induced inhibition of cancer cell growth via induction of p21WAF1 expression.

In conclusion, results of the present study significantly accelerated our understanding of the molecular mechanisms of decursin; cell proliferation, apoptosis, cell cycle progress, and the signaling pathway in bladder and colon cancer cells. In particular, the novel aspect of these results is that the decursininduced inhibition of cell growth is apparently associated with ERK activation via the expression of p21WAF1. Thus, the results of the present study demonstrate that decursin is useful for the treatment of bladder and colon cancer.

\section{Acknowledgements}

This work was supported by a Korea Science and Engineering Foundation (KOSEF) grant funded by the Korean government (MEST) (R11-2008-014-01001).

\section{References}

1. Chinery R, Brockman JA, Peeler MO, Shyr Y, Beauchamp RD and Coffey RJ: Antioxidants enhance the cytotoxicity of chemotherapeutic agents in colorectal cancer: a p53independent induction of p21WAF1/CIP1 via C/EBPbeta. Nat Med 3: 1233-1241, 1997.

2. Koenig F, Jung K, Schnorr D and Loening SA: Urinary markers of malignancy. Clin Chim Acta 297: 191-205, 2000.

3. Lee S, Shin DS, Kim JS, Oh KB and Kang SS: Antibacterial coumarins from Angelica gigas roots. Arch Pharm Res 26: 449-452, 2003

4. Sarker SD and Nahar L: Natural medicine: the genus Angelica. Curr Med Chem 11: 1479-1500, 2004

5. Kim HH, Sik Bang S, Seok Choi J, Han H and Kim IH: Involvement of PKC and ROS in the cytotoxic mechanism of anti-leukemic decursin and its derivatives and their structureactivity relationship in human K562 erythroleukemia and U937 myeloleukemia cells. Cancer Lett 223: 191-201, 2005.

6. Ahn KS, Sim WS and Kim IH: Decursin: a cytotoxic agent and protein kinase $\mathrm{C}$ activator from the root of Angelica gigas. Planta Med 62: 7-9, 1996.

7. Son SH, Kim MJ, Chung WY, Son JA, Kim YS, Kim YC, Kang SS, Lee SK and Park KK: Decursin and decursinol inhibit VEGF-induced angiogenesis by blocking the activation of extracellular signal-regulated kinase and c-Jun N-terminal kinase. Cancer Lett 280: 86-92, 2009.

8. Lee S, Lee YS, Jung SH, Shin KH, Kim BK and Kang SS: Antitumor activity of decursinol angulated and decursin from Angelica gigas. Arch Pharmacol Res 26: 727-730, 2003.

9. Yim D, Singh RP, Agrwal C, Lee S, Chi H and Agarwal R: A novel anticancer agent, decursin, induces $\mathrm{G}_{1}$ arrest and apoptosis in human prostate carcinoma cells. Cancer Res 65: 1035-1044, 2005.

10. Moon SK, Jung SY, Choi YH, Lee YC, Patterson C and Kim CH: PDTC, metal chelating compound, induces G1 phase cell cycle arrest in vascular smooth muscle cells through inducing p21Cip1 expression: involvement of p38 mitogen activated protein kinase. J Cell Physiol 198: 310-323, 2004.
11. Hu W and Kavanagh JJ: Anticancer therapy targeting the apoptotic pathway. Lancet Oncol 4: 721-729, 2003.

12. Yang J, Liu X, Bhalla K, Kim CN, Brado AM, Cai J, Peng TI, Jones DP and Wang X: Prevention of apoptosis by Bcl-2: release of cytochrome c from mitochondria blocked. Science 275: 1129-1132, 1997

13. Nicholson DW, Ali A, Thornberry NA, Thornberry JP, Ding CK, Gallant M, Gareau Y, Griffin PR, Labelle C, Lazebnik YA, Munday NA, Raju SM, Smulson ME, Yamin TT, Yu VL and Miller DK: Identification and inhibition of the ICE/CED-3 protease necessary for mammalian apoptosis. Nature 376: 37-43, 1995.

14. Lazebnik YA, Kaufmann SH, Desnoyers S, Poirier GG and Earnshaw WC: Cleavage of poly(ADP-ribose) polymerase by a proteinase with properties like ICE. Nature 371: 346-347, 1994.

15. Tewari M, Quan LT, O'Rourke K, Desnoyers S, Zeng Z, Beidler DR, Poirier GG, Salvesen GS and Dixit VM: Yama/CPP32 beta, a mammalian homolog of CED-3, is a CrmA-inhibitable protease that cleaves the death substrate poly(ADP-ribose) polymerase. Cell 81: 801-809, 1995 .

16. Harada $\mathrm{H}$ and Grant S: Apoptosis regulators. Rev Clin Exp Hematol 7: 117-138, 2003.

17. Degli Esposti M and Dive C: Mitochondrial membrane permeabilisation by Bax/Bak. Biophys Res Commun 304: 455-461, 2003.

18. Cory S, Huang DC and Adams JM: The Bcl-2 family: roles in cell survival and oncogenesis. Oncogene 22: 8590-8607, 2003.

19. Gross A, Mcdonnell JM and Korsmeyer SJ: Bcl-2 family members and the mitochondria in apoptosis. Genes Dev 13: 1899-1911, 1999.

20. Smaili SS, Hsu YT, Sanders KM, Russell JT and Youle RJ: Bax translocation to mitochondria subsequent to a rapid loss of mitochondrial membrane potential. Cell Death Differ 8: 909-920, 2001.

21. Hsu YT, Wolter KG and Youle RJ: Cytosol-to-membrane redistribution of Bax and $\mathrm{Bcl}-\mathrm{XL}$ during apoptosis. Proc Natl Acad Sci USA 94: 3668-3672, 1997.

22. Kluck RM, Bossy-Wetzel E, Green DR and Newmeyer DD: The release of cytochrome $\mathrm{c}$ from mitochondria: a primary site for Bcl-2 regulation of apoptosis. Science 275: 1132-1136, 1997.

23. Harnois C, Demers MJ, Bouchard V, Vallee K, Gagne D and Fujit N, Tsuruo T, Vézina A, Beaulieu JF, Côté A and Vachon PH: Human intestinal epithelial crypt cell survival and death: complex modulations of Bcl-2 homologs by Fak, PI3-K/Akt-1, MEK/Erk, and p38 signaling pathways. J Cell Physiol 198: 209-222, 2004

24. Weinberg RA: The retinoblastoma protein and cell cycle control. Cell 81: 323-330, 1995.

25. Sherr CJ: Cancer cell cycles. Science 274: 1672-1677, 1996

26. Cai K and Dynlacht BD: Activity and nature of p21(WAF1) complexes during the cell cycle. Proc Natl Acad Sci USA 95: 12254-12259, 1998.

27. Sherr CJ and Roberts JM: Inhibitors of mammalian G1 cyclindependent kinases. Gene Dev 9: 1149-1163, 1995.

28. Davis RJ: The mitogen-activated protein kinase signal transduction pathway. J Biol Chem 268: 14553-14556, 1993.

29. Cobb M and Goldsmith EJ: How MAP Kinases are regulated. J Biol Chem 270: 14843-14846, 1995.

30. Davis RJ: MAPKs: new JNK expands the group. Trends Biochem Sci 19: 470-473, 1994

31. Xia Z, Dickens M, Raingeaud J, Davis RJ and Greenberg ME: Opposing effects of ERK and JNK-p38 MAP kinases on apoptosis. Science 270: 1326-1331, 1995. 